\title{
Intracranial Extension
}

National Cancer Institute

\section{Source}

National Cancer Institute. Intracranial Extension. NCI Thesaurus. Code C153176.

The direct spread of a malignant tumor from an adjacent site into the cranium. 